\title{
Estimation of the number of people in an indoor environment based on WiFi received signal strength indicator
}

\author{
Hakam M. Zaidan, Emad A. Mohammed, Dheyaa H. Alhelal \\ Department of Computer Technology Engineering, Technical Engineering College/Mosul, Northern Technical University, \\ Iraq
}

\begin{tabular}{l} 
Article Info \\
\hline Article history: \\
Received Sep 29, 2020 \\
Revised Apr 27, 2021 \\
Accepted May 17, 2021
\end{tabular}

Keywords:

LOS

Non-LOS

Number of people

RSSI

WiFi

\begin{abstract}
WiFi access points are widely spread everywhere in all our daily life routines. Using these devices to provide services other than the Internet is becoming familiar nowadays. This paper conducts an experimental study to estimate the number of people in an indoor environment through two system setups, line of sight, and non-line of sight. Relationship modeling between WiFi received signal and the number of people uses polynomial regression. The experiment comprised of two stages: first is the data collection from a controlled number of people. Then, the collected data used to train the system through polynomial regression. The second is testing the system's effectiveness by applying it to an uncontrolled environment. Testing results revealed efficiency in using WiFi received signal strength to do the people counting (up to 60) because of the accuracy achievements of $93.17 \%$ in the line of sight system. The non-line of sight system disclosed randomness in the received signal strength indicator regardless of the change in the number of people. The randomness is mainly caused by the fading effect of the concrete wall. Therefore it is inefficient to use the non-line of sight system in concrete buildings.
\end{abstract}

This is an open access article under the CC BY-SA license.

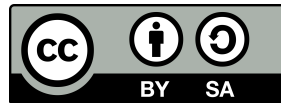

\section{Corresponding Author:}

Hakam M. Zaidan

Department of Computer Technology Engineering

Technical Engineering College/Mosul

Northern Technical University, Mosul/Iraq

Email: hakam_m_zaidan@ntu.edu.iq

\section{INTRODUCTION}

In the past two decades, the use of the Internet has grown dramatically. The Internet has become one of the necessities of modern life. In the current time, it is hard to find a building or floor in that building does not have $\mathrm{WiFi}$ [1]. It has become natural to take advantage of these devices (WiFi) and use them to provide services other than providing the Internet, such as estimating the number of people in that area. In different situations, it is necessary to know the number of people in a specific area to control the number or to improve the services [2]. The traditional way to count the number of people in many cases is complicated; and requires additional hardware, which makes it expensive especially if the number of people is related to doing something automatically, or in real-time applications. Researchers were implemented the people counting in different methods. Some of them used cameras for the counting process as in [3]. While the authors in [4] did that depending on sensors. Further, the researchers in [5] used devices carried by people such as smartphones or 
RFID to estimate the number of people.

Regularly, the WiFi signal is subjected to many variations through its way to the receiver depending on multiple factors, starting with the distance between transmitter (router) and receiver, furniture in the way between, and other obstacles, including humans. In our case, estimating the number of people is intended toward closed rooms such as conference or lecture rooms inside universities. Because the furniture (in such indoor places) is constant, the substantial factor that affects the WiFi signal, in this case, is the people inside the room.

In this work, we proposed two system setups to inspect in the estimation of the people count. The system setups are line of sight (LOS) and non-line of sight (non-LOS). Both systems are examining the WiFi received signal strength indicator (RSSI) from the router installed in the room. The utilization of the existing WiFi access point is an efficient way to serve our purpose and requires no additional cost. In the LOS system, the transmitting and the receiving stations were placed in the same room, while in the non-LOS system, we removed the receiver station to a neighboring room. In this case, there is no direct line of sight between the transmitter and the receiver stations. The non-LOS system is considered in this paper to investigate the effect of eliminating the direct line-of-sight path between the transmitter and the receiver on the people counting process. In other words, in the non-LOS environment, the received signal will be only the superposition of various copies of the WiFi signal from diverse paths (multipath fading) without any direct line-of-sight. Thus, the received signal will be strongly affected by obstacles between the transmitter and the receiver, including the people. The two methods, LOS and non-LOS, were proposed to investigate which one is more accurate than the other in the estimation process. An empirical study is applied to do the estimation. The study used the already installed WiFi access point as the transmitter. The NodeMCU development board, which contains ESP8266 WiFi SOC is used as a receiver to conduct the RSSI examination and data collection [6].

The relationship between RSSI and obstacles is not perfectly linear. Therefore, polynomial regression is one of the best ways to model this kind of data distribution. Thus, polynomial regression is going to be used to model the relationship between the independent variable (in our case, the RSSI) and the dependant variable (the number of people) as the $\mathrm{n}$ th degree polynomial. We are going to reveal which polynomial degree that best fits the RSSI data distribution in which underfitting, as well as overfitting, will be avoided.

Many procedures are used to estimate the number of people in a specific area. Some of those procedures are used extra hardware in the estimation process, such as cameras, sensors, and smartphones [7]-[9]. Additional hardware adds additional cost to the estimation process. There exist another procedures that depends on using WiFi to estimate the number of people as our case in this paper [10]-[20], all of them lack accuracy, and used a small number of people in testing. The authors in [21] proposed a framework to estimate the number of people in a specific area depending on the concept that the transmitted signal carries the signature of people, in other words, blocking the Line of Sight and Propagation effects. After putting these two concepts together, they developed a mathematical expression to describe the relationship between the amplitude of the received signal and the total number of occupants. The paper used a small number of people to test (maximum of 9 people) in the meanwhile, the error rate is increasing as the number of people increasing. The authors in [22] were able to estimate the number of people by taking the received signal and analyze its Doppler spectrum. They realized that any increase in the crowd density increases the Doppler spectrum peaks; again, the testing on this approach took place on a small number of people (maximum of 7). Also, the crowd density accuracy is not high enough. Here another technique is used [23], where the researchers depend on the inter-event times to count the total number of people. Their framework shows how the inter-event times have significant information that enabled them to estimate the number of people in a specific area. The authors tested their framework with more than 40 experiments in different areas, and they got results with high accuracy, but again they used a small number of people (a maximum of 9). The researchers in [24] used the kNN classifier. To do the training, they used one transmitting and two receiving nodes, and they collected RF data to form a database that consists of the features. In the end, the authors used the Bootstrap re-sampling technique to optimize the time required for training and to extract an accurate feature; this work achieved 94\% accuracy with a maximum of 18 people. The authors in [2] used linear regression to estimate the people count; they achieved $77.2 \%$ accuracy in only seven people. To summarize our work:

- The proposed method does not require people to carry any devices (no phone, sensors, or transmitters), which makes it affordable and easier to implement.

- The proposed method is tested for a large number of people (up to 60), while other works used only 7,9, and 18 people. 
- The proposed method does not require prior analysis, such as feature extractions before the data is fed into the main regression model.

- The proposed method achieved high accuracy in comparison to similar research works.

The rest of the paper is structured as follows. Section 2 explains the research method, including the system setup, data collection, and estimation modeling. Section 3 presents the results obtained from the training and the testing of our system. Finally, section 4 states the conclusion.

\section{RESEARCH METHOD}

\subsection{Overview}

Usually, RSSI is affected by objects, moving and stationary, between the transmitter and the receiver. These objects cause reflection, diffraction, and scattering. Also, the multipath and the distance between the transmitter and the receiver affects signal strength significantly. Whenever the distance increases, the signal attenuation increases, which leads to signal degradation. The same applies to the number of people because they have effects, like other objects, on signal degradation; in other words, the more people in the room, the more impact on signal degradation. For example, the expected RSSI value when there are 5 people in a room is larger than the RSSI value when there are 20 people in the same room.

During an event in the intended room, the cumulative distribution function (CDF) of the RSSI was measured in two environments, LOS and non-LOS. The reason behind those measurements is to study the feasibility of people counting depending on WiFi RSSI. The CDFs were measured by collecting multiple RSSI measures from the intended router via the proposed receiver system. The obtained results revealed a strong relationship between the number of people and the RSSI value. The results of this test are explained in detail in the results section.

The experiment will take place in two stages: the first one is the data collection from a controlled environment (controlling the number of people inside the room). Then, the previously collected data used to train the system through polynomial regression. The second stage is testing the system's effectiveness by applying it in an uncontrolled environment.

\subsection{Environment and system setup}

Regarding the system test environment, the project was applied in the conference room at the Technical College, Mosul-Iraq. Table 1 shows the simulation environment parameters. In this work, the distance variations are not the case because there is a fixed distance of $15 \mathrm{~m}$ between the WiFi access point and the receiver station.

Table 1. Simulation environment parameters

\begin{tabular}{lc}
\hline Room area & $15 \mathrm{~m} \times 8 \mathrm{~m}$ \\
\hline Capacity & 60 people \\
Construction type & Concrete \\
Transmitter & TP-Link Router (TL-WR841N) \\
Receiver & NodeMCU microcontroller \\
Furniture & Whiteboard, projector, Wooden Table, \\
& an iron door, 60 iron seats, and 6 split type AC devices \\
\hline
\end{tabular}

Two system setups were considered LOS and non-LOS (shown in Figure 1). The already installed WiFi access point was used as the transmitter, while the NodeMCU is used as the receiver to collect the RSSI data. We have chosen the NodeMCU because it is an open-source, interactive, programmable, low cost, low power, and WiFi-enabled that is easily used to prototype IoT products. The NodeMCU collects the RSSI data from the already installed WiFi access point in the intended room. Then, the NodeMCU makes the people number estimation based on the collected RSSI information. To evaluate our system effectiveness, we set up our system in the conference room at the intended room during regular daily work hours. The receiver system is programmed to take the RSSI readings for 10 seconds and calculate the average, then apply the proposed estimation algorithms. 


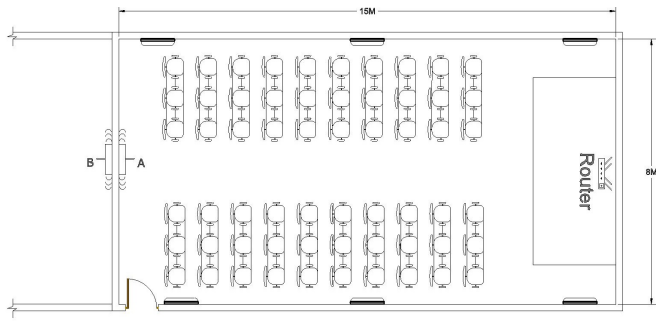

Figure 1. Layout of the conference room in the technical college, Mosul-Iraq; area: $15 \mathrm{~m} \mathrm{x} \mathrm{8m;} \mathrm{contains} 60$ seats. (A: Receiver in LOS system setup; B: Receiver in non-LOS system setup)

\subsection{Data collection}

From previous results, the relationship between RSSI value and the number of people needs to be studied. Experimental results were conducted to examine the relationship between RSSI and the number of people. The study was started by placing the receiver and the transmitter at the same height in both systems. Then, the experiments took place on ten sets of people. The first set includes five people, the second set the number of people increased to ten, and so on. In each set, the number of people increases by five people until the tenth set, we had fifty. In other words, the number of people is increased gradually from 5 to 50 , starting from the first set that includes five people only, ending with the tenth set that has fifty.

In each set, we advised the people to sit in 10 random sitting positions. In this way, we are trying to mimic the randomness of people sitting as much as possible. In each sitting position, the RSSI value is measured ten times, the average of these ten readings is considered. To this point, there are ten average readings for each set of people, one average reading per sitting position. Eventually, one average RSSI value has been considered as an aggregate of those ten averages (an overall average). In other words, for each set of people, there are 100 RSSI values, everything regarding this matter is clarified in Figure 2.

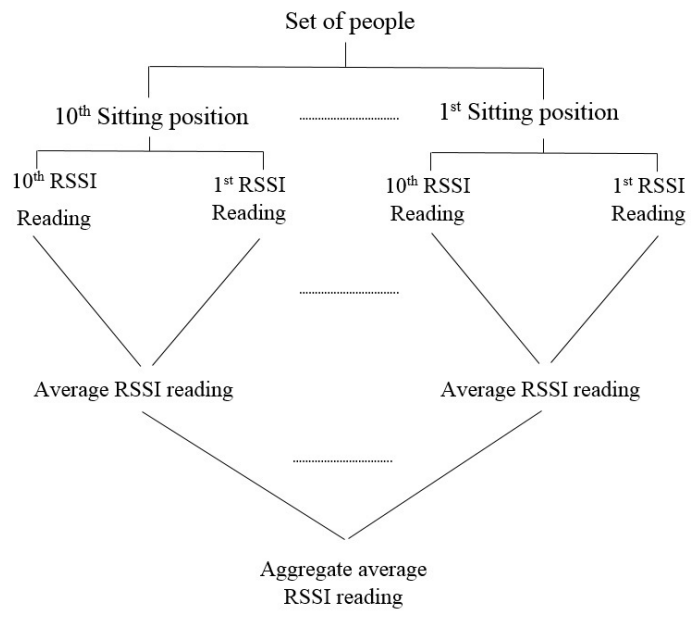

Figure 2. Set of people with 10 random sitting positions; each position has an average RSSI, and then an aggregate average will correspond to the number of people in that set

\subsection{Estimation modeling}

RSSI data obtained empirically is going to be used in the estimation training using polynomial regression. Polynomial regression was used because of the non-linear behavior between the independent variable (RSSI) and the dependent variable (number of people). First, second, and third-degree polynomials, (1) from [25], were applied in the estimation modeling process.

$$
\psi=\alpha_{0}+\alpha_{1} x+\alpha_{2} x^{2}+\alpha_{3} x^{3}+\ldots+\alpha_{n} x^{n}+\epsilon
$$

where $\alpha_{0,1,2,3, \ldots, n}$ are the polynomial coefficients, $x$ is the RSSI (independent variable), $\psi$ is the estimated number of people (dependent variable), $n$ is the $\mathrm{n} t h$ degree polynomial, and $\epsilon$ is an unobserved random error with a zero mean conditioned on the independent variable $x$. 
To calculate the total error rate regarding our model, we have to compute it based on the difference between the estimated and the real number of people. In (2) is used to calculate the total number of errors.

$$
\text { TotalErrorRate }=\frac{\sum_{n=1}^{N} \frac{\left|R_{n}-E_{n}\right|}{R_{n}}}{N}
$$

where $R_{n}$ is the real number of people currently in the room, $E_{n}$ is the estimated number of people, and $N$ is the total number of investigated cases.

\section{RESULT AND DISCUSSION}

Figure 3 a shows the CDF of the LOS environment. The figure shows that the RSSI values are ranging from a maximum RSSI value of $-31 \mathrm{dBm}$ to a minimum value of $-72 \mathrm{dBm}$ over time. These variations are due to reflection, diffraction, and scattering in addition to fading. The aforementioned attributes are emerging from moving and stationary objects between the transmitter and the receiver. The LOS CDF shows that approximately $34 \%$ of the time the router operates in the RSSI region between $-30 \mathrm{dBm}$ and $-60 \mathrm{dBm}$. While $66 \%$ of the time the router operates in less than $-60 \mathrm{dBm}$. Also, only $4 \%$ of the time the router operates at the minimum RSSI value.

Figure $3 \mathrm{~b}$ shows the CDF of the non-LOS environment. The figure shows that the RSSI values are ranging from $-33 \mathrm{dBm}$ as the maximum RSSI to a minimum of $-75 \mathrm{dBm}$ over time. The effect of reflection, diffraction, scattering, and fading were increased in the non-LOS environment. This affection is due to the addition of more obstacles, a concrete wall in this case, between the transmitter and the receiver. As can be noticed from non-LOS CDF is that approximately $29 \%$ of the time the router operates in the RSSI region between $-33 \mathrm{dBm}$ and $-60 \mathrm{dBm}$. While $71 \%$ of the time the router operates in less than $-60 \mathrm{dBm}$. Also, only $8 \%$ of the time the router operates at the minimum RSSI value.

Figures 3 show that the people counting is possible as the RSSI value ranging between up and down during the change in the number of people. The minimum received RSSI values were $4 \%$ and $8 \%$ of the time for the LOS and non-LOS respectively. In our case, since the furniture and other obstacles are stationary between the transmitter and the receiver, the fluctuations in RSSI value mainly depend on the variations in the number of people. In other words, the number of people in the room is changing, and the room is not always full of people.



(a)

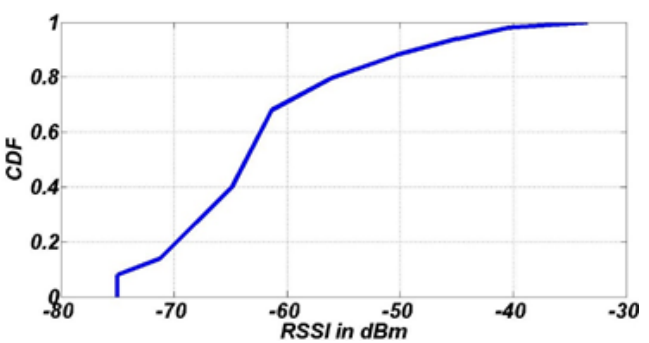

(b)

Figure 3. Cumulative distribution function (CDF) of RSSI. It can be observed that the RSSI is changing over time which means that the number of people has an impact on RSSI. The figure shows (a) LOS environment,

(b) non-LOS environment

Regrading the non-LOS system, the obtained results revealed a large margin of fluctuation and randomness. For example, the average RSSI obtained when there were 35 people in the room was $-49 \mathrm{dBm}$, while when there were 20 people in the room, the average RSSI was $-50 \mathrm{dBm}$ (Figure 4). The same applies when there were 5, and 15 people in the room. It seemed that the concrete wall fading effect on the RSSI has a very random impact on the RSSI value and carries lots of noise. Behavior like this holds a large amount of randomness is unpredictable, makes it very hard to model, and yields a noticeable error rate. For this reason, we decided not to go far with the non-LOS system. 


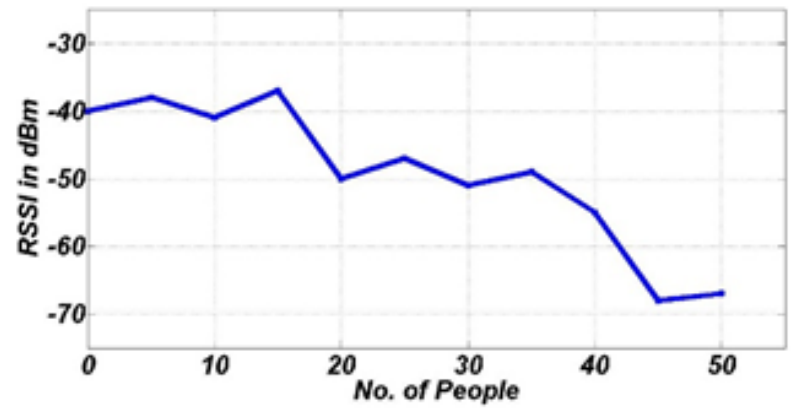

Figure 4. non-LOS system results, the concrete wall adds a noticeable amount of noise to the RSSI

Figure 5 shows the obtained results from the LOS system that we are going to use in the system training process. Results showed a slight change in the RSSI in each set of people. This change is due to many reasons, for example, the people moving in their seats, and the movement of the fans that are circulating air in the room. Moreover, the results showed a noticeable change in the RSSI when the number of people is changing. An important matter that worths mentioning is the overlap among RSSI values when the number of people is changing. For example, the overlap in RSSI when there were 15, 20 people in the room, and when there were 30,35 people in the room. The effect of this kind of overlap was reduced by taking the average of the RSSI values that belong to each set of people.

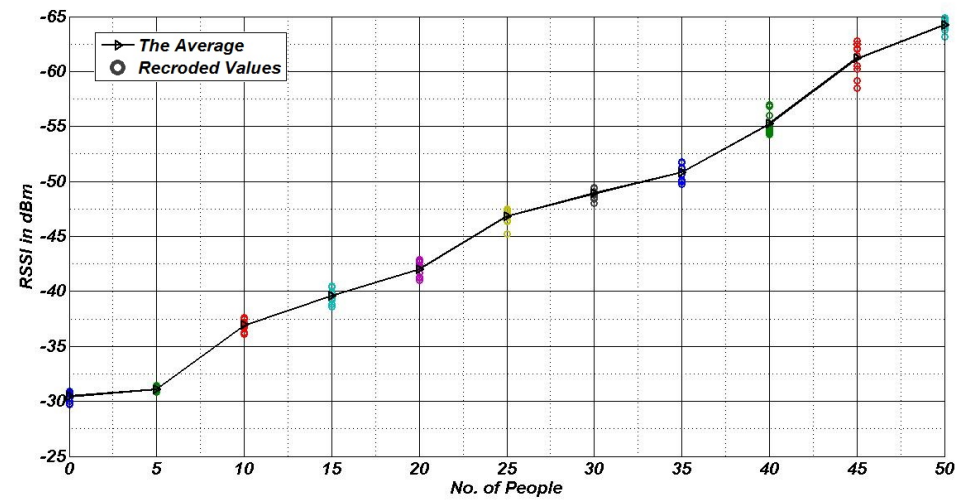

Figure 5. Obtained results, from a controlled number of people in LOS environment. A noticeable change in the RSSI is clear when the number of people is changing

Figure 6 shows the system performance for a period of two regular consecutive workdays during events that took place in the conference room. The figure is organized to express the ground truth with the expected values represented by different polynomial degrees. Also, it includes the difference between the ground truth and the estimated value represented by each polynomial degree. This difference explains the degree of accuracy provided by each polynomial degree. The more the difference is closer to zero, the more accurate and precise estimation acquired. The obtained estimation accuracy is as the following: the $1^{\text {st }}$ degree polynomial revealed an accuracy of $90.5 \%$, the $2^{\text {nd }}$ degree polynomial presented an accuracy of $92.08 \%$, and the $3^{\text {rd }}$ order polynomial gave an accuracy of $93.17 \%$. We had tested further polynomial degrees as the following: $4^{\text {th }}, 5^{\text {th }}, 6^{\text {th }}$, and $7^{\text {th }}$-degree. The acquired accuracies were $93.27 \%, 92.59 \%, 91.1 \%$, and $90 \%$ respectively. The obtained accuracy from the $4^{t h}$-degree polynomial revealed a very slight enhancement if compared to the $3^{\text {rd }}$-degree polynomial, while the accuracy began to decrease whenever the polynomial degree increases. Therefore, we stopped at the $3^{r d}$-degree polynomial as our final way of people estimation because it has the higher accuracy, and to avoid overfitting.

Table 2 shows a comparison between our model and similar research works. We compared the accuracy of our proposed method against the accuracy of other methods presented within the last five years. The accuracy of some of the methods in the table was originally presented accuracy as an error in the number of 
people. For easier comparison, we normalized the accuracies so that they are all in terms of percentages. The accuracy of our proposed method is higher than four out of five approaches that we compared against. The $\mathrm{kNN}$ classifier method has higher accuracy than our proposed method but with a less number of people. It used only 18 people, while we utilized 60 people to calculate the accuracy. In the case of 21 people, we achieved an accuracy of $\simeq 99 \%$ (Figure 6).

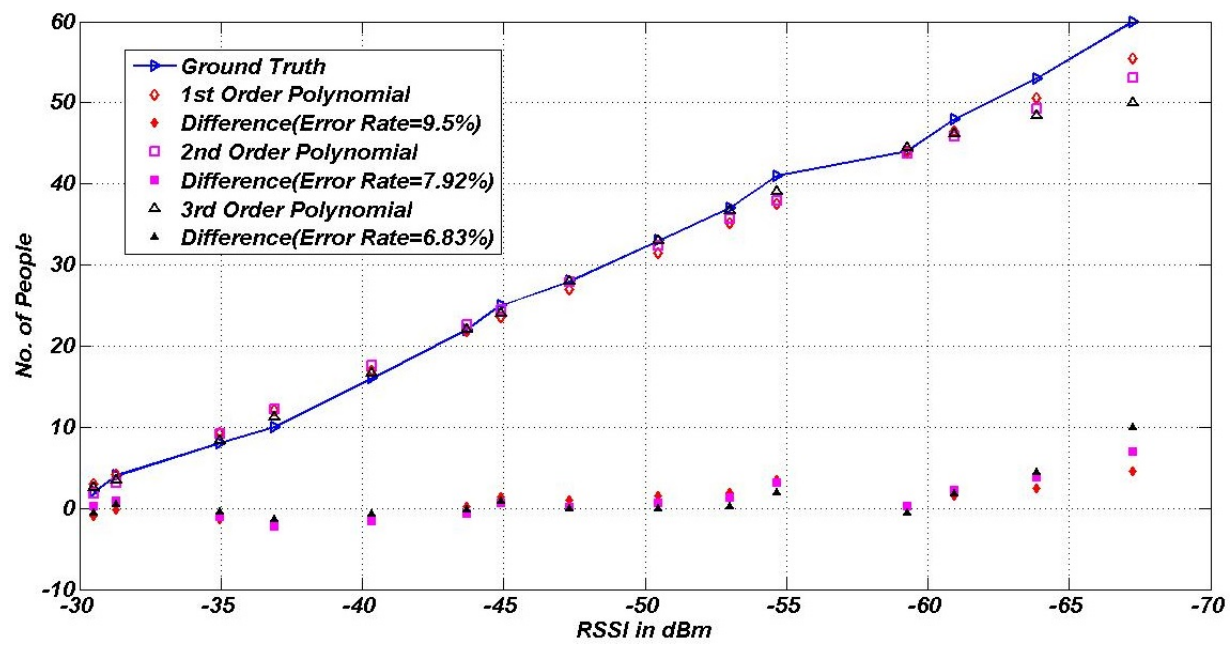

Figure 6. System evaluation; the estimated number of people vs the real number; the difference belongs to polynomial 3 is the nearest to zero in most of the cases which means that it has the best accuracy

Table 2. Our proposed approach vs. Similar research works

\begin{tabular}{lccc}
\hline Ref & Accuracy & No. of People & Way of Modeling \\
\hline Our proposed method & $93.27 \%$ & 60 & Polynomial regression \\
{$[2]$} & $77.2 \%$ & 7 & Linear regression \\
{$[21]$} & $77.7 \%$ & 9 & Mathematical expression with Kullback-Leibler divergence \\
{$[22]$} & $86 \%$ & 7 & Doppler spectrum analysis \\
{$[23]$} & $77.7 \%$ & 9 & PMF of the inter-event times \\
{$[24]$} & $94 \%$ & 18 & kNN classifier \\
\hline
\end{tabular}

To ensure that our system can be applied to different environments, we ran a system test in a different scenario. We moved our proposed system to a lecture hall in another building in the college. The tests ran in three classes: the first one has 15 people, the second one has 21 people, and the third one contains 31 people. Each test ran for two hours. The system is programmed to provide an estimation every 15 minutes. Test results (Figure 7) revealed the robustness of our proposed system as results showed an accuracy of \pm 1 person in the three cases.

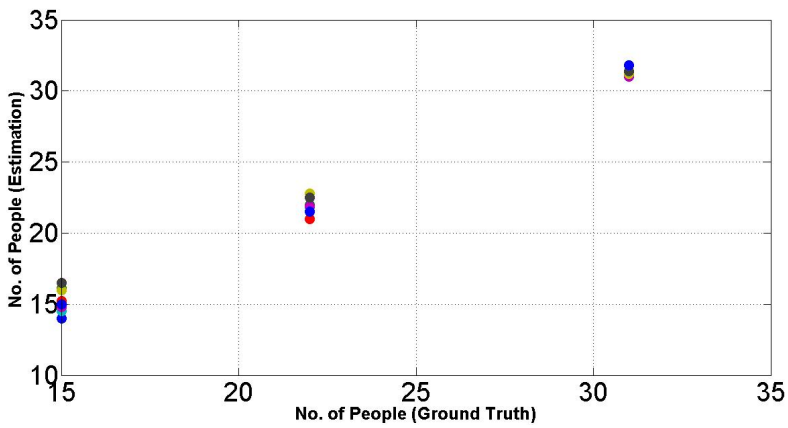

Figure 7. Ground truth vs estimation; three tests, the first one includes 15 people, the second one includes 21 people, and the last one includes 31 people ( \pm 1 person is the error rate) 


\section{CONCLUSION}

In this work, two people counting strategies are studied, LOS and non-LOS systems. The non-LOS system seemed to fluctuate, a little unpredictable, and showed a high margin of error. On the contrary, the LOS system results showed robustness, a predictable counting strategy, and an acceptable margin of error. Because of the nonlinear relationship between RSSI and the number of people, polynomial regression revealed a high accuracy in system modeling. We achieved our main goals in this paper. The first goal is the low-cost people counting strategy that suits real-time applications. The second goal is to use a large number of people in the training process (50) and the estimation process (60). The LOS system showed a high degree of accuracy in the estimation of the number of people in an indoor environment, which makes it applicable in real-time applications. As future research, we are planning to use our people counting method in real-time applications.

\section{REFERENCES}

[1] D. Singla, H. Singh, J. Chawla, S. Ranjan, and S. Parvanda, ”Wi-fi signal strength indicator," 2016.

[2] T. Yoshida and Y. Taniguchi, "Estimating the number of people using existing wifi access point in indoor environment," in Proceedings of the 6th European Conference of Computer Science (ECCS'15), 2015, pp. 46-53.

[3] Z. Ma and A. B. Chan, "Crossing the line: Crowd counting by integer programming with local features," in Proceedings of the IEEE Conference on Computer Vision and Pattern Recognition, Portland, OR, USA, 2013, pp. 25392546, doi: 10.1109/CVPR.2013.328.

[4] S. Fujii, Y. Taniguchi, G. Hasegawa, and M. Matsuoka, "Pedestrian counting with grid-based binary sensors based on monte carlo method," in Springer Plus,vol. 3, no. 1, p. 299, 2014, doi: 10.1186/2193-1801-3-299

[5] L. Schauer, M. Werner, and P. Marcus, "Estimating crowd densities and pedestrian flows using wi-fi and bluetooth," in Proceedings of the 11th International Conference on Mobile and Ubiquitous Systems: Computing, Networking and Services, 2014, pp. 171-177, doi: 10.4108/icst.mobiquitous.2014.257870.

[6] L. K. P. Saputra and Y. Lukito, "Implementation of air conditioning control system using REST protocol based on NodeMCU ESP8266, " 2017 International Conference on Smart Cities, Automation Intelligent Computing Systems (ICON-SONICS) 2017, pp. 126-130, doi: 10.1109/ICON-SONICS.2017.8267834.

[7] S. Fujisawa, G. Hasegawa, Y. Taniguchi, and H. Nakano, "Pedestrian counting in video sequences based on optical flow clustering," International Journal of Image Processing, vol. 7, no. 1, pp. 1-16, 2013, doi. 10.1.1.735.4072.

[8] Y. Taniguchi and H. Nakano, "Modeling and evaluation of a ceiling-mounted compound-eye sensor,"International Information Institute (Tokyo). Information, vol. 17, no. 2, p. 663, 2014.

[9] Y. Wang, J. Yang, H. Liu, Y. Chen, M. Gruteser, and R. P. Martin, "Measuring human queues using wifi signals," in Proceedings of the 19th annual international conference on Mobile computing networking, 2013, pp. 235-238, doi: $10.1145 / 2500423.2504584$.

[10] R. Zhou, X. Lu, Y. Fu, and M. Tang, "Device-free crowd counting with wifi channel state information and deep neural networks," Wireless Networks, pp. 1-12, 2020, doi: 10.1007/s11276-020-02274-7.

[11] S. Liu, Y. Zhao, F. Xue, B. Chen, and X. Chen, "Deepcount: Crowd counting with wifi via deep learning, ” arXiv preprint arXiv:1903.05316, 2019.

[12] C. Groba, "Demonstrations and people-counting based on wifi probe requests," 2019 IEEE 5th World Forum on Internet of Things (WF-IoT) IEEE, 2019, pp. 596-600, doi: 10.1109/WF-IoT.2019.8767208.

[13] O. T. Ibrahim, W. Gomaa, and M. Youssef, "Crosscount: A Deep Learning System for Device-Free Human Counting Using WiFi," in IEEE Sensors Journal, vol. 19, no. 21, pp. 9921-9928, 1 Nov 2019, doi: 10.1109/JSEN.2019.2928502.

[14] U. Mehmood, I. Moser, P. P. Jayaraman, and A. Banerjee, "Occupancy estimation using wifi: A case study for counting passengers on busses," in 2019 IEEE 5th World Forum on Internet of Things (WF-IoT), Limerick, Ireland, 2019, pp. 165-170, doi: 10.1109/WF-IoT.2019.8767350.

[15] O. Oshiga, H. U. Suleiman, S. Thomas, P. Nzerem, L. Farouk, and S. Adeshina, "Human detection for crowd count estimation using csi of wifi signals," in 2019 15th International Conference on Electronics, Computer and Computation (ICECCO), Abuja, Nigeria, 2019, pp. 1-6, doi: 10.1109/ICECCO48375.2019.9043195.

[16] S. Kianoush, S. Savazzi, V. Rampa, and M. Nicoli, "People counting by dense wifi mimo networks: Channel features and machine learning algorithms," Sensors, vol. 19, no. 16, p. 3450, 2019, doi: 10.3390/s19163450.

[17] U. Singh, J.-F. Determe, F. Horlin, and P. De Doncker, "Crowd forecasting based on wifi sensors and LSTM neural networks," in IEEE Transactions on Instrumentation and Measurement, vol. 69, no. 9, pp. 6121-6131, Sept. 2020, doi: 10.1109/TIM.2020.2969588.

[18] W. Xi, et al., "Electronic frog eye: Counting crowd using wifi, " IEEE INFOCOM 2014-IEEE Conference on Computer Communications. IEEE, 2014, pp. 361-369, doi: 10.1109/INFOCOM.2014.6847958.

[19] Y. Yang, J. Cao, X. Liu, and X. Liu, "Wi-count: Passing people counting with cots wifi devices, " in 2018 27th International Conference on Computer Communication and Networks (ICCCN), Hangzhou, China, 2018, pp. 1-9, 
doi: 10.1109/ICCCN.2018.8487420.

[20] H. Zou, Y. Zhou, J. Yang, W. Gu, L. Xie, and C. Spanos, "Freecount: Device-free crowd counting with commodity WiFi, ”in GLOBECOM 2017-2017 IEEE Global Communications Conference, Singapore, 2017, pp. 1-6, doi: 10.1109/GLOCOM.2017.8255034.

[21] S. Depatla, A. Muralidharan, and Y. Mostofi, "Occupancy estimation using only wifi power measurenments," IEEE Journal on Selected Areas in Communications, vol. 33, no. 7, pp. 1381-1393, July 2015, doi: 10.1109/JSAC.2015.2430272.

[22] S. Di Domenico, G. Pecoraro, E. Cianca, and M. De Sanctis, ”Trained-once device-free crowd counting and occupancy estimation using wifi: A doppler spectrum based approach, 2016 IEEE 12th International Conference on Wireless and Mobile Computing, Networking and Communications (WiMob), New York, NY, USA, 2016, pp. 1-8, doi: 10.1109/WiMOB.2016.7763227.

[23] S. Depatla and Y. Mostofi, "Crowd counting through walls using wifi, "in 2018 IEEE International Conference on Pervasive Computing and Communications (PerCom), Athens, Greece, 2018, pp. 1-10, doi: 10.1109/PERCOM.2018.8444589.

[24] M. Taha, R. Atallah, O. Dwiek, and F. Bata, "Crowd estimation based on rssi measurements using knn classification, "in 2020 3rd International Conference on Intelligent Autonomous Systems (ICoIAS), Singapore, 2020, pp. 67-70, doi: 10.1109/ICoIAS49312.2020.9081850.

[25] E. Ostertagova, "Modelling using polynomial regression," Procedia Engineering," vol. 48, pp. 500-506, 2012, doi: 10.1016/j.proeng.2012.09.545. 